\title{
Application of Reverse Anterolateral Thigh Flap in Infected Knee Defect Reconstruction in a Pediatric Patient with Developmental Disability
}

\author{
Won Ha, MD ${ }^{1}$ Kyu Nam Kim, MD² Chae Ri Park, BSc
}

Chi Sun Yoon, $\mathrm{MD}^{2}$
Address for correspondence Chi Sun Yoon, MD, Department of Plastic and Reconstructive Surgery, Ulsan University Hospital, University of Ulsan College of Medicine, 877, Bangeojinsunhwando-ro, Dong-gu, Ulsan 682-714, South Korea (e-mail: ycscem@uuh.ulsan.kr).

Development of the microsurgical technique and knowledge of vascular anatomy has led to a high success rate and good results in pediatric free flap reconstruction. However, in free flap reconstruction in pediatric patients, there is difficulty in flap dissection and vessel anastomosis because of the small size of the vessels and the tendency of vasospasm. ${ }^{1,2}$ In addition, in uncooperative patients with mental and developmental disability, disastrous results can occur postoperatively.

Reconstruction of the soft tissue defect of the knee remains difficult and challenging and becomes even more difficult in defects accompanied by infection. This is because local available tissues are often unhealthy and not sufficient to cover the large defect. ${ }^{3,4}$ Some reconstructive options have been introduced so far, including local muscle flap, reverse anterolateral thigh (ALT) flap, and free flap. In the free flap, major vessels are usually located deeply, requiring meticulous and tedious dissection which can result in injury to major vessel and requiring a long operation time. ${ }^{3,5,6}$ Also, with a local muscle flap, there is relevant donor-site morbidity including contour deformity. ${ }^{3}$

ALT flap, one of the most popular flaps, has been used as a pedicled or free flap with a wide variety of clinical applications. ${ }^{5-8}$ Despite some reports on efficacy of reconstruction using ALT free flap in pediatric foot and ankle defects, $^{2}$ few cases of reverse ALT flaps successfully transferred for reconstruction of soft tissue defects of the knee have been reported. Therefore, we reconstructed an infected soft tissue knee defect in a pediatric patient, who had no cooperation capability due to mental and developmental disability, using reverse ALT flap and report the case.

received

June 11, 2015

accepted after revision

July 23, 2015

published online

January 21, 2016

\section{Case}

A 13-year-old adolescent boy with history of seizure and mental and developmental disability developed dermatitis on the entire body and a wound on the knee region arising from a fingernail scratch.

This uncooperative child had irritated his knee wound with continuous scratching and infection had developed. This child presented to our emergency department and knee joint aspiration was performed. Morganella morganii spp. was found in the fluid. He was treated with antibiotics in the pediatric department and referred to our department.

First, incision and drainage was performed. Serial irrigation and debriding the devitalized and infected tissue was performed to control the infection, and negative pressure wound therapy was applied for 6 weeks. Once infection was controlled, we evaluated the final defect around the knee and decided on reconstruction with a reverse ALT flap. Based on preoperative computed tomography (CT) angiography, there was a connection between the lateral femoral circumflex vessel and superior lateral genicular vessel. The flap was designed to be larger than the original defect due to characteristic tight skin of pediatric patients. The size of the flap was $11 \times 10 \mathrm{~cm}$. In the pedicle dissection, the proximal descending branch of the lateral femoral circumflex vessel was clamped with a microclamp before division to check for the existence of strong pulsation of reverse pedicle and venous flap congestion; after verifying the vascular perfusion of the distally based flap, the proximal descending branch was divided and dissection of the distal descending branch with preservation of motor nerve was performed. To prevent vasospasm and instability of patella by weakening the vastus lateralis muscle, we dissected the pedicle to the site where Tel: +1(212) 584-4662.
Copyright $\odot 2016$ by Thieme Medical Publishers, Inc., 333 Seventh Avenue, New York, NY 10001, USA.

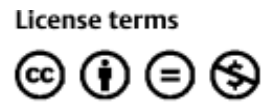

$10.1055 / \mathrm{s}-0035-1570536$ ISSN $2377-0813$. 
the pedicle enters vastus lateralis muscle and insetted the flap carefully so as not to pull. After checking the flap circulation, the flap was fixed carefully to avoid compression of the pedicle, and the donor site was covered with skin graft. The postoperative course for this uncooperative patient was uneventful, and the flap survived completely. No recurrence of wound infection was observed at the 1-year follow-up (-Figs. 1-3).

\section{Discussion}

Free flap reconstruction of knee soft tissue defect is also considered challenging in pediatric patients as well as adult patients because recipient vessel selection was difficult due to deep-lying major vessel. ${ }^{3}$ In pediatric patients, besides the aforementioned difficulty in selection of a recipient vessel, the free flap becomes even more difficult because of smallscale vessel and small-scale surrounding tissue. ${ }^{1}$ Furthermore, flap failure can occur postoperatively in uncooperative patients with mental and developmental disability.

Serial irrigation and debridement is imperative for management of an infected wound. As a result, defects become larger after complete debridement of infected tissue. In cases of infected defect reconstruction, ${ }^{4}$ well-vascularized muscle flaps have generally been accepted as a more useful flap than

A
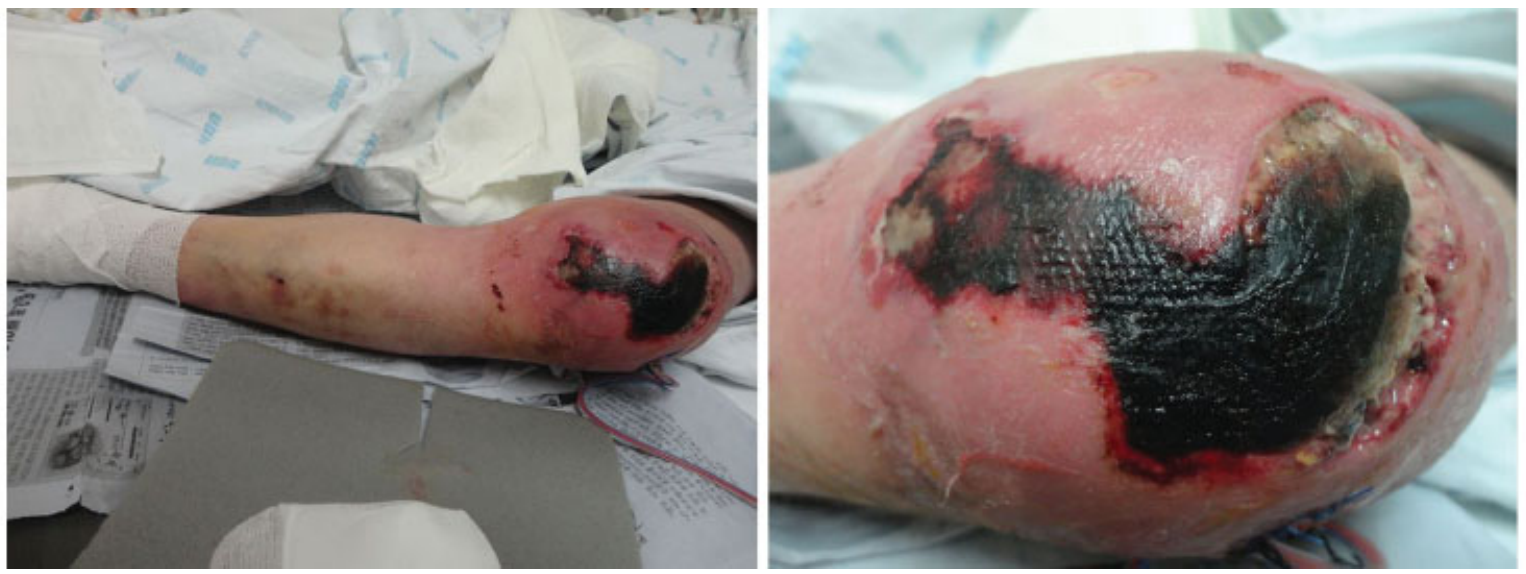

Fig. 1 A, B Infectious wound on the knee region arising from a fingernail scratch.
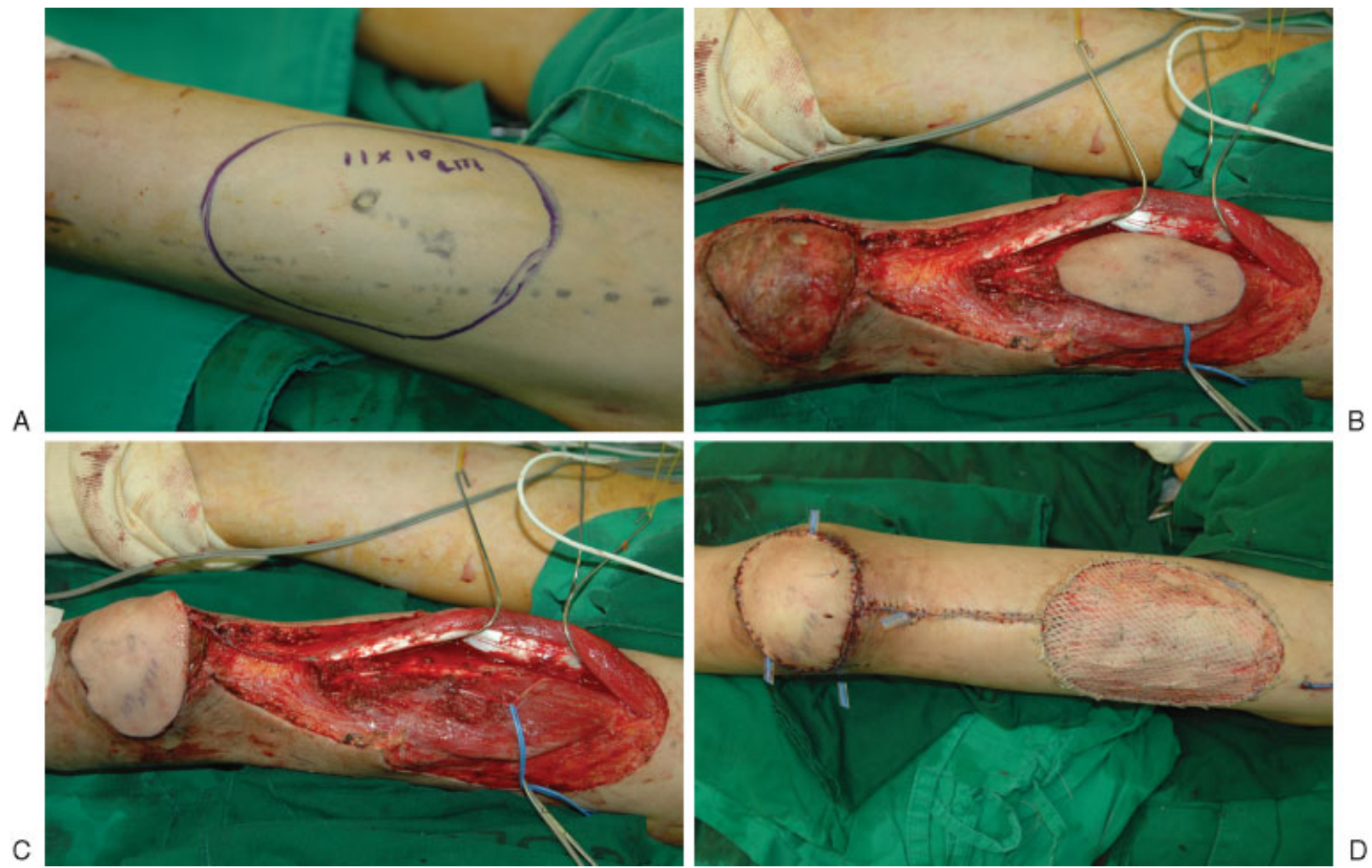

Fig. 2 (A) The design of the anterolateral thigh perforator flap. (B) We dissected the pedicle to the site where the pedicle enters vastus lateralis muscle. (C) We insetted the flap carefully so as not to pull. (D) After distally based ALT flap. 
44 Reverse ALT Flap in Pediatric Patient Ha et al.

A

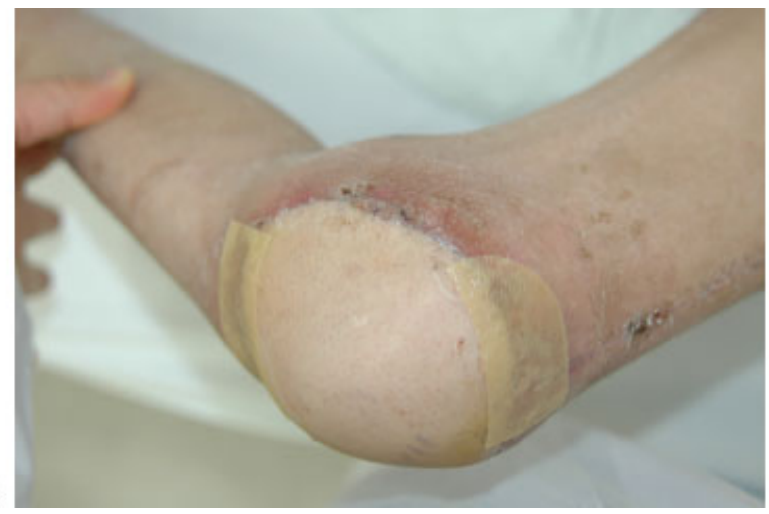

Fig. 3 A, B Three weeks after the operation.

fasciocutaneous flap. ${ }^{4}$ However, muscle flap can cause donorsite morbidity and contour deformity. Particularly in growing pediatric patients, donor-site morbidity is more significant than in adult patients. Therefore, we reconstructed an infected soft tissue knee defect with reverse ALT flap in a pediatric patient with developmental disability. Since the first description of reverse ALT flap in 1990, it has been used in reconstruction of knee soft tissue defect. ${ }^{5,6}$ It has several advantages, including lower donor-site morbidity, no loss of muscle function or sacrifice of major arteries, a wider surface, and greater arc of rotation. However, some limitations have been reported, including a wide range of anatomical variation and venous congestion. ${ }^{5,6}$ To overcome reported disadvantages, we performed preoperative evaluation of vessel status with $\mathrm{CT}$ angiography to check the connection between the lateral femoral circumflex vessel and superior lateral genicular vessel. In addition, before dividing the proximal descending branch of the lateral femoral circumflex vessel, a vascular clamp was used to temporarily occlude the proximal blood supply and the necessity of further venous supercharging was checked.

The patient did not cooperate postoperatively and could not maintain absolute bed resting position. Hence, we conducted hourly monitoring to prevent flap loss and maintain immobilization through medical procedures until the 10th postoperative day. Fortunately, the flap survived completely.

\section{Conclusion}

We suggest that the reverse ALT flap is an adequate reconstructive option in cases of infectious knee soft tissue defect

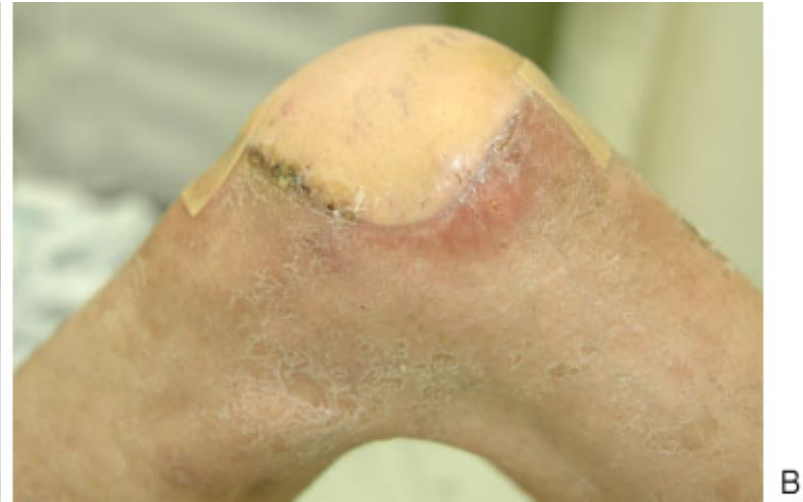

in uncooperative pediatric patients, under close observation for flap monitoring and patient immobilization.

\section{Conflicts of Interest}

None.

\section{References}

1 Song JW, Ben-Nakhi M, Hong JP. Reconstruction of lower extremity with perforator free flaps by free style approach in pediatric patients. J Reconstr Microsurg 2012;28(9):589-594

2 Acar MA, Güleç A, Aydin BK, Erkoçak ÖF, Yilmaz G, Şenaran H. Reconstruction of foot and ankle defects with a free anterolateral thigh flap in pediatric patients. J Reconstr Microsurg 2015;31(3): 225-232

3 Hong JP, Koshima I. Using perforators as recipient vessels (supermicrosurgery) for free flap reconstruction of the knee region. Ann Plast Surg 2010;64(3):291-293

4 Kim SW, Hwang KT, Kim JD, Kim YH. Reconstruction of postinfected scalp defects using latissimus dorsi perforator and myocutaneous free flaps. J Craniofac Surg 2012;23(6):1615-1619

5 Lin F, Wong M, Chew KY, Tan BK. Incorporating a racket-handle skin bridge and venous supercharging to improve reliability of the distally based anterolateral thigh flap. Arch Plast Surg 2015;42(2): 240-241

6 Erba P, Raffoul W, Bauquis O. Safe dissection of the distally based anterolateral thigh flap.J Reconstr Microsurg 2012;28(6):405-411

7 Bhadkamkar MA, Wolfswinkel EM, Hatef DA, et al. The ultra-thin, fascia-only anterolateral thigh flap. J Reconstr Microsurg 2014; 30(9):599-606

8 Nosrati N, Chao AH, Chang DW, Yu P. Lower extremity reconstruction with the anterolateral thigh flap. J Reconstr Microsurg 2012; 28(4):227-234 